\title{
The occurrence of Entamoeba gingivalis among patients with periodontal disease
}

\author{
JoAnna LuszczaK ${ }^{1 *}$, Michal BartosiK ${ }^{1}$, Jolanta Rzymowska ${ }^{1}$, \\ Agnieszka Sochaczewska-Dolecka ${ }^{2}$, Ewa Tomaszek ${ }^{3}$, \\ JoAnna Wysokinska-MiszCZUK ${ }^{3}$, ANna Bogucka-KockA ${ }^{1}$
}

${ }^{1}$ Chair and Department of Biology and Genetics Medical University of Lublin, Chodzki 4a, 20-093 Lublin, Poland ${ }^{2}$ Chair and Department of Conservative Dentistry Medical University of Lublin, Karmelicka 7, 20-093 Lublin, Poland ${ }^{3}$ Department of Periodontology Medical University of Lublin, Karmelicka 7, 20-093 Lublin, Poland

\section{ARTICLE INFO}

Received 12 January 2016

Accepted 02 March 2016

\section{Keywords:}

Entamoeba gingivalis, periodontal disease,

oral cavity,

public health.

\begin{abstract}
According to some studies, the Entamoeba gingivalis colonizing the gingival tissue is an important agent in bringing about periodontitis. Other studies, however, deem it an opportunist that is able to survive in the medium induced by periodontal disease. The aim of this study was to investigate the prevalence of Entamoeba gingivalis infection in patients from the Department of Periodontology, and compare this population with that of healthy people, so as to analyze the relationships between infection and patient sex and age. The result of this work is that in both groups, a correlation $(p=0,19)$ has been noted between the occurrence of amoebae and other diseases in the oral cavity. Indeed, 81,4\% of all patients with some periodontal disease showed the presence of amoeba. Among those who are not afflicted with oral diseases, the presence of amoeba was indicated in $62,5 \%$ of the total. In addition, a correlation between the person's age and the presence of protozoa $(\mathrm{p}=0,15)$ was strongly marked among women $(\mathrm{p}=0,19)$. In the three age groups of women in this study (40-49, 60-69, and above 80 years), we observed a $100 \%$ presence of protozoa.

Our study leads us to the conclusion that infections with Entamoeba gingivalis should be regarded as an factor that is associated with the pathological changes occurring in patients with periodontal diseases.
\end{abstract}

\section{INTRODUCTION}

Entamoeba gingivalis is considered by some authors to be a pathogenic protozoan, and to be commensal by others. It colonizes the gingival tissue and was the first amoeba described in humans [2]. Entamoeba gingivalis is the only species of amoeba that can phagocytose nuclear fragments of ingested bacteria, cellular debris, leucocytes and epithelial cells and occasional red blood cells - all of which are often seen within the large, numerous food vacuoles [10,12]. Their spheroid nucleus is rounded with a delicate central (like Entamoeba histolytica) and is a big karyosome (similar to Entamoeba coli). Moreover, the nuclear membrane is lined with coarse chromatin granules. Morphologically, this amoeba resembles Entamoeba histolytica, and diagnosis requires great attention. Entamoeba gingivalis has a large number of pseudopodia that allow it to move quickly. Furthermore,

\footnotetext{
* Corresponding author

e-mail: joanna.barczak@umlub.pl
}

it does not have resistant forms (cysts) recognizable in the clinical specimens, but only trophozoites, which vary in size from 10 to $35 \mu \mathrm{m}[2,5,11]$.

This parasite inhabits the area around the teeth and gums, including the spaces between teeth, and especially in teeth cavities, dental tartar, necrotic mucosa of cells and the gingival fringes of the gums $[11,17]$.

Entamoeba gingivalis is found in the healthy oral cavity as a scavenger [10]. According to some studies, this amoeba is also an important agent in the induction of periodontitis. Still other researchers consider it merely an opportunist capable of surviving in the medium induced by periodontal disease $[11,17]$. It is known, however, that Entamoeba gingivalis cooperates with synergistic, symbiotic bacteria in engendering periodontal disease in hosts with low immunity [8]. What is more, E. gingivalis is recognized as causing gum itch, fatigue, halitosis, severe headaches, sore palate and periodontal tissue damage [11]. 
Many researchers have found that the infection rates of this amoeba in patients with periodontitis are higher than those of healthy control groups; thus they inferred that its colonization was related to periodontitis $[3,6,13,14]$. The presence of these organisms in the mouth correlates with poor oral hygiene, and they are quite common among people who have a low standard of life, contrary to the notion that parasites avoid the oral cavity [4].

According to some studies, E. gingivalis produces a particular, progressive, periodontal disease among immunocompromised patients $[7,15]$. A Swedish study showed its association with human immunodeficiency virus type 1 (HIV-1) and reported that no other parasite was ever found in this type of immunodeficiency [15].

The diagnosis of Entamoeba gingivalis is still based on direct microscopy of tissue scrapings or on preculture in specialized parasitological media [4].

As there are controversies on the correlation of this amoeba to periodontal diseases, in the following study, we intended to determine the prevalence of Entamoeba gingivalis in patients with periodontal diseases and in healthy people. We also attempted to assess the correlation between the frequency of Entamoeba gingivalis colonization and patient sex and age.

\section{MATERIALS AND METHODS}

Diagnostic material in the form of swabs was collected from 102 patients with periodontal disease, who were undergoing treatment in the Department of Periodontology of the Medical University of Lublin. The same was undertaken with 32 people assessed as being healthy. Among all 134 specimens, 88 were taken from women and 46 from men.

The samples from the gingival pouch, subgingival areas and gums were obtained by using sterile swabs, and then were examined for the presence of Entamoeba gingivalis. The trophozoites were detected via microscopic preparations $(400 \times)$ made not longer than 30 minutes after the collection of samples.

In so-doing, the sample swabs were first washed in $0,9 \%$ $\mathrm{NaCl}$. A drop of extract was then put on a clean slide to which a drop of $0.1 \%$ safranin solution was added in order to make the parasites' cell morphology visible and to facilitate their counting (Photo. 1, 2).

Entamoeba gingivalis cells were identified by observation of morphology and the characteristic movement of their pseudopodia. Next, the correlation between the prevalence of E. gingivalis infection and the patients' sex and age was calculated.

All analyses were performed with the use of Statistica ${ }^{\circledR} 10$ software for Windows.

\section{RESULTS}

The study involved 134 persons: 88 women and 46 men. In the studied group, 102 people had some periodontal disease, while the remaining 32 patients were healthy. The study consisted of the observation of samples derived by way of buccal swabs under a microscope in a search for Entamoeba gingivalis trophozoites (Photo. 1, 2). Such protozoa were found in 103 patients $(77 \%)$, but not in 31 patients (23\%) (Fig. 1). Among the samples derived from women, the protozoa were in 68 (77\%), and among men, in 35 subjects $(77 \%)$. No correlation was seen between gender and the presence or absence of protozoa $(\mathrm{P}=0,06)$.

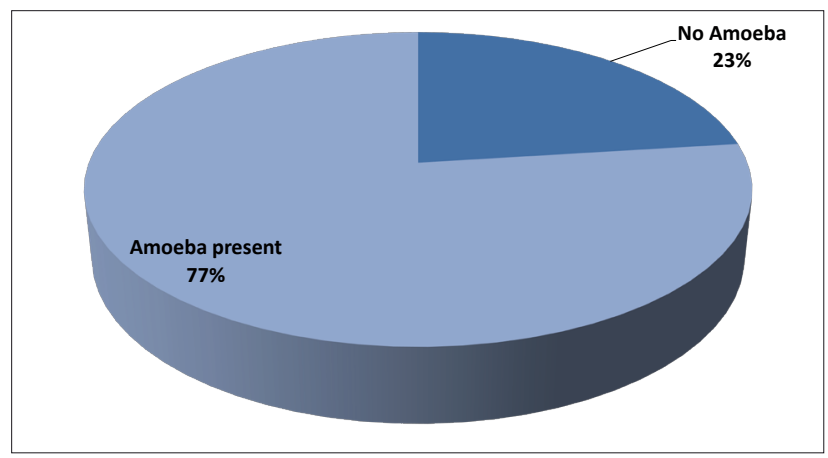

Figure 1. Specific characteristics of the Entamoeba gingivalis present in all patients

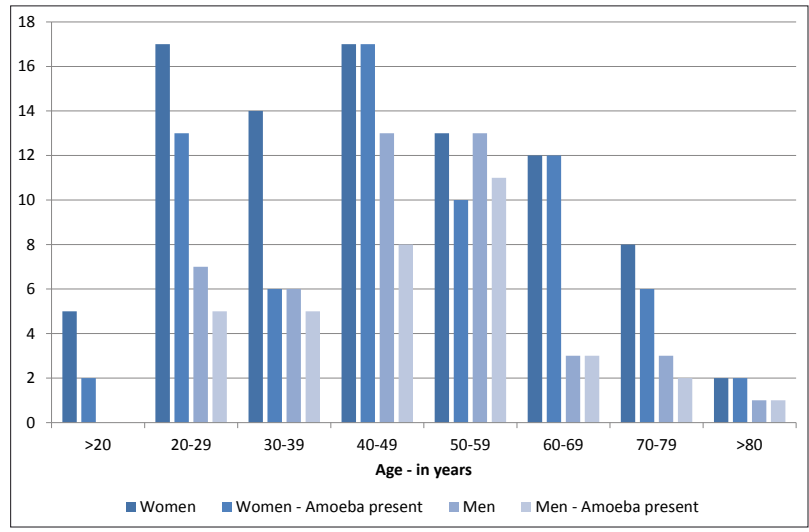

Figure 2. Categorization according to the age

The age of respondents ranged from 18 to 81 years - the average age was 46,1 years ( $\mathrm{SD}=16,62$ years), for women; $-49,5$ years $(\mathrm{SD}=17,88$ years $)$ for men and 46,39 years ( $\mathrm{SD}=14,06$ years) combined. A selected group was characterized by near normal decomposition (KS test $d=0,085$ ). The correlation between the person's age and the presence of protozoa $(\mathrm{p}=0,15)$, was strongly marked among women $(\mathrm{p}=0,19)$, but it could not be observed in men $(\mathrm{P}=0,06)$. In the three age groups of women (40-49, 60-69, and above 80 years -31 patients in total), $100 \%$ presence of protozoa was observed. Among men, the protozoa was observed in only two groups of age (60-69 and above 80 years) and they were much fewer in number (only four patients) (Fig. 2).

In both groups, a correlation $(p=0,19)$ between the occurrence of amoebae and other diseases in the oral cavity have been noted. Regarding patients with some periodontal disease (102 individuals), 83 (81\%) showed the presence of amoeba. This protozoa was not found in only 19 persons (19\%) (Fig. 3). By sex, this constitutes, for women (in total, a group of 68 patients), 55 individuals (82\%); and for men (in total, a group of 35 patients), 28 persons (80\%). Among those who did not have oral diseases (31 cases), the amoeba was evident in $20(65 \%)$. By sex, this constitutes for women (a population of 21$), 13$ persons (62\%); and for men (a population of 35$), 28$ people $(80 \%)$. 

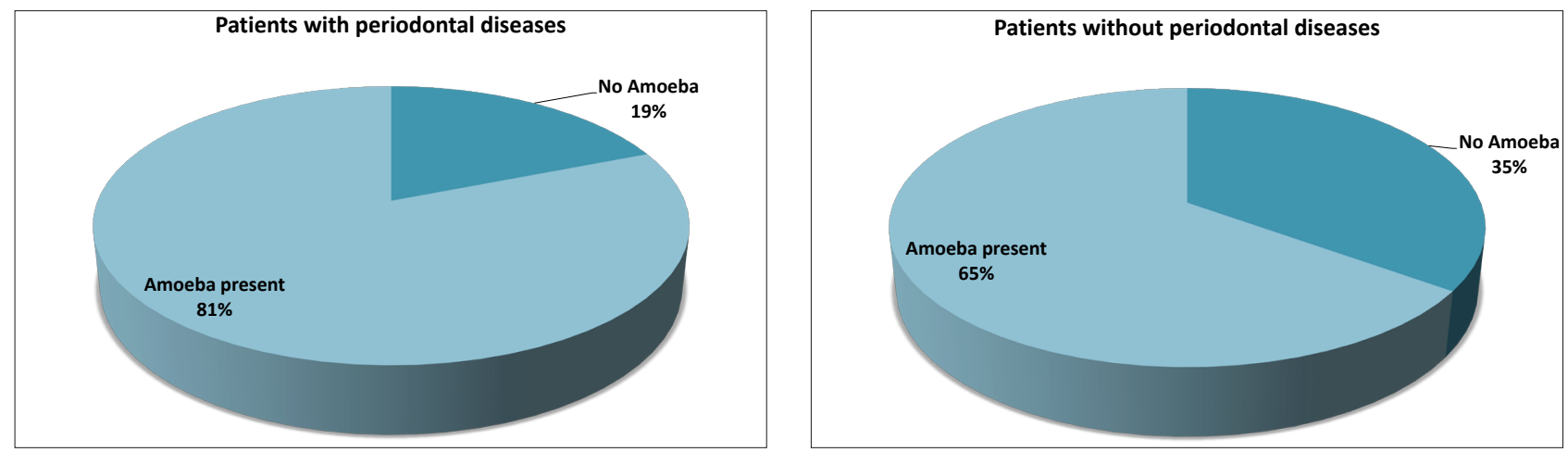

Figure 3. Correlation between the occurrence of amoebae and other periodontal diseases

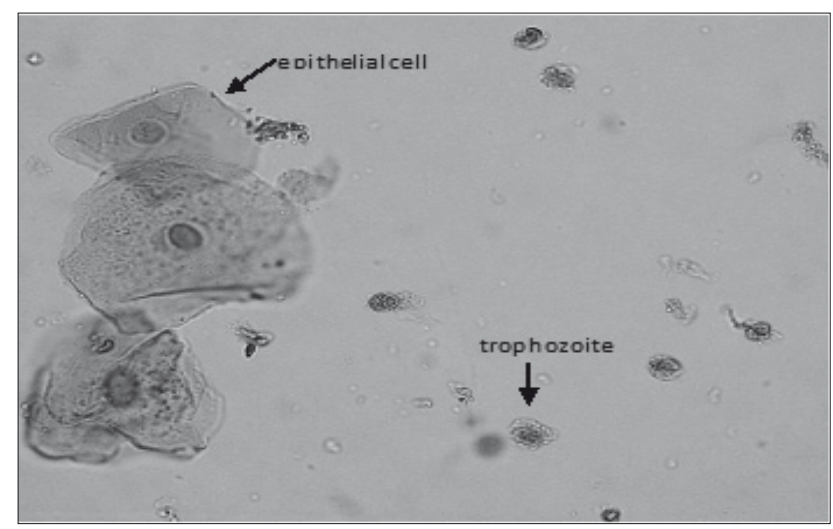

Photo 1. Trophozoites of Entamoeba gingivalis safranin stained (200x)

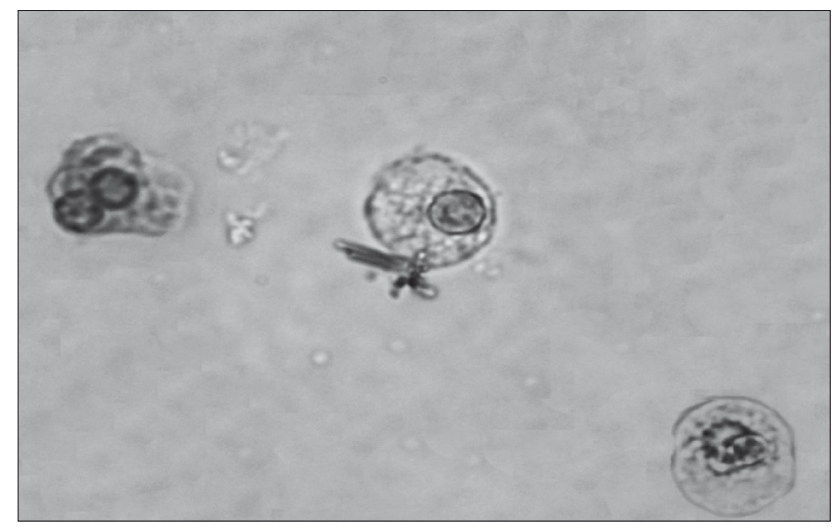

Photo 2. Trophozoites of Entamoeba gingivalis safranin stained (1000×)

\section{DISCUSSION}

Infectious parasites are categorized into those causing local infections, and those inducing systemic infections with indirect effects. However, saprophytes such as Entamoeba gingivalis (which belongs to the first group), have the potential to turn into opportunistic pathogens, or freeliving amoebas that occasionally become invasive $[1,4]$.

The activity of $E$. gingivalis in oral cavity disease lesion progression is associated with a capacity for erythrocytophagy and the presence of enzymes important in the development of periodontitis. Lyon and workers described the possible pathogenic mechanism of Entamoeba gingivalis. Herein, the leukocyte metabolism altered by amoebas and the excessive release of elastase brought about the destruction of the periodontium, and it induced gingival bleeding. Furthermore, the access of amoeba to the erythrocytes enables the neutralization of oxidants generated in the inflammatory process and the maintenance of the anaerobic conditions that are optimal for the amoeba $[9,16]$.

In our study, the high prevalence rate of Entamoeba gingivalis is correlated to periodontal tissue condition. In both studied groups, we have noted the correlation $(p=0,19)$ between the occurrence of amoebae and other diseases in the oral cavity. Indeed, $81,4 \%$ of all patients with some periodontal disease showed the presence of amoeba. Among those who did not have oral diseases, this amoeba occurred in $62,5 \%$ of the total. Of note, a correlation between the person's age and the presence of protozoa $(p=0,15)$, was strongly marked among women $(p=0,19)$. In the three age groups of women who took part in this study, (40-49, 60-69, and above 80 years), 100\% presence of protozoa was observed.

Many workers have found that the infection rates of Entamoeba gingivalis in patients with periodontitis were higher than those of healthy groups. Thus, they inferred that this amoeba is an important cause of periodontal disease, generating gum itch, sore palate, halitosis, fatigue, severe headaches, and periodontal tissue damage $[2,11,18]$. It seems that following proper oral hygiene instructions is essential in the control of this infection. Moreover, the occurrence of E. gingivalis is correlated with the age of the host, so oral protozoa are rarely found in children, while the frequency of infection increases with age [18].

In our study, a more common occurrence of Entamoeba gingivalis was found in patients with pathological changes in the oral cavity than in healthy individuals. In addition, our microscopic observations has led us to consider that infection with Entamoeba gingivalis should be regarded as a factor associated with the pathological changes occurring in patients with periodontal diseases. The results also suggest that this technique is applicable to the detection and identification of E. gingivalis in the human oral cavity. $E$. gingivalis invasion of the periodontium and the role of this protozoan in the periodontal disease process merit further investigation and cooperation between dental clinics and laboratories in diagnosing parasitic infections [11]. 


\section{CONCLUSIONS}

Our study has led us to the conclusion that infections with Entamoeba gingivalis should be regarded as an factor associated with the pathological changes occurring in patients with periodontal diseases.

\section{REFERENCES}

1. Albuqerque R.L., et al.: Incidence of Entamoeba gingivalis and Trichomonas tenax in samples of dental biofilm and saliva from patients with periodontal disease. Rev. Gaucha. Odontol., 59, 35-40, 2011.

2. Al-Saeed W.M.: Pathogenic effect of Entamoeba gingivalis on gingival tissue of rats. Al-Rafidain. Dent. J., 3, 70-73, 2003.

3. Al-Saeed W.M., Mahmood H.J.: Prevalence of Entamoeba gingivalis in dental patients in Mosul. Al-Rafidain. Dent. J., 1, 65-68, 2001.

4. Bergquist R.: Parasitic infections affecting the oral cavity. Periodontol., 49, 96-105, 2000.

5. Bhaijee F., Bell D.: Entamoeba gingivalis in Acute Osteomyelitis of the Mandible. Case. Rep. Med., 2011.

6. Bonner M., et al.: Detection of the amoeba Entamoeba gingivalis in periodontal pockets. Parasite., 21-30, 2014.

7. Cembranelli S.B., et al.: First evidence of genetic intraspecific variability and occurrence of Entamoeba gingivalis in HIV(+)/AIDS. PloS One., 8, 12, 2013.

8. Chen J.F., et al.: Studies on periodontal disease caused by Entamoeba gingivalis and its pathogenetic mechanism. CMJ., 114, 12-15, 2001.
9. Chomicz L., et al.: Amoebae transmitted into the human oral cavity and their role in the development of pathological changes - on the basis of literature. Czas. Stomatol., 5, 321-329, 2007.

10. Foda A.A., El-Malky M.M.: Prevalence of genital tract infection with Entamoeba gingivalis among copper T 380A intrauterine device users in Egypt. Contraception., 85, 108-12, 2012.

11. Gharavi MJ., et al.: Buccal cavity Protozoa in patients referred to the Faculty of Dentistry in Tehran, Iran. IJP., 1, 43-46, 2006.

12. Jian B, et al.: Entamoeba gingivalis pulmonary abscess - diagnosed by fine needle aspiration. Cytopathology J., 2, 1-3, 2008.

13. Lin G.Y., Chen J.F., Ma W.M.: Investigation of infection of oral protozoan of parts of persons in Fuzhou, Strait. AJPM., 3, 129-130, 1997.

14. Linke H.A., Gannonn J.T., Obin J.N.: Clinical survey of Entamoeba gingivalis by multiple sampling in patients with advanced periodontal disease. Int. J. Parasitol., 19, 803-808, 1989.

15. Lucht E, et al: Entamoeba gingivalis in human immunodeficiency virus type 1-infected patients with periodontal disease. Clin. Infec. Dis., 27, 471-473, 1999.

16. Lyon, T., et al.: Oral amoebiasis: the role of Entamoeba gingivalis in periodontal disease. Quintessence Int., 12, 1245-1248, 1983.

17. Ponce de León P., et al.: Relation between buccal protozoa and $\mathrm{pH}$ salivary IgA in patients with dental prothesis. Rev. Inst. Med. Trop. Sao Paulo., 43, 241-242, 2001.

18. Sarowska J., et al.: The Occurrence of Entamoeba gingivalis and Trichomonas tenax in patients with periodontal diseases, immunosuppression and genetic diseases. Adv. Clin. Exp. Med., 13, 291-297, 2004. 